\title{
Otimização da suspensão para obtenção de cerâmicas porosas via "gelcasting"
}

\section{(Suspension optimization in order to obtain porous ceramics through "gelcasting")}

\author{
M. O. Carlos, I. R. Oliveira, J. R. Garcia, V. C. Pandolfelli \\ Departamento de Engenharia de Materiais - DEMa \\ Universidade Federal de S. Carlos - UFSCar \\ Rod. Washington Luiz, km 235, C.P.676, S. Carlos, SP, 13565-905 \\ marianaoc@polvo.ufscar.br,vicpando@power.ufscar.br
}

\begin{abstract}
Resumo
Processos envolvendo altas temperaturas consomem grandes quantidades de energia, influenciando o custo final de produtos. Assim, uma necessidade emergente na área de cerâmicas refratárias é o desenvolvimento de materiais isolantes para altas temperaturas. Esses materiais devem apresentar poros pequenos, serem pouco permeáveis, apresentar adequada resistência mecânica e baixa retração volumétrica quando em uso. Portanto, esse trabalho teve como objetivo avaliar a influência do método de produção da suspensão nas propriedades de cerâmicas porosas obtidas por meio da incorporação de uma espuma aquosa. Esta avaliação foi realizada utilizando-se medidas de porosidade, retração linear e resistência mecânica. Os resultados obtidos mostraram que a otimização da suspensão pode ser obtida pela escolha adequada da matéria-prima, bem como do teor de sólidos, resultando em cerâmicas com maior porosidade aliada a adequada resistência mecânica.

Palavras-chave: cerâmicas porosas, isolantes para altas temperaturas, "gelcasting".
\end{abstract}

\begin{abstract}
Processes involving high temperatures consume great amounts of energy, influencing the final cost of the products. Therefore, there is a clear market need for the development of refractory ceramics with insulating properties for high temperatures application. These materials should present small pores, low permeability, suitable mechanical resistance and low volumetric shrinkage. In this work, the influence of the suspensions in the properties of porous ceramic, obtained by foam incorporation into the ceramic suspension, was evaluated through porosity, linear shrinkage and mechanical strength measurements. The results showed that the appropriate choice of raw materials, as well as solids content of the suspension, resulted in larger porosity. Such aspects should provide porous ceramic with higher mechanical strength due to their superior strut density.
\end{abstract}

Keywords: porous ceramic, insulating for high temperatures, gelcasting.

\section{INTRODUÇÃO}

Um problema comum nos processos industriais, especialmente aqueles que usam altas temperaturas, é a necessidade de reduzir perdas energéticas.

Propriedades intrínsecas aos materiais cerâmicos, como inércia química, refratariedade e resistência à abrasão, associadas a algumas características próprias de sólidos celulares, como alta área superficial, permeabilidade e baixa condutividade térmica, permitem que as cerâmicas porosas possam contribuir para a redução dos altos gastos de energia. Esses materiais são insubstituíveis em várias aplicações como filtros para purificação de gases, de metais líquidos e isolamento térmico em temperaturas não suportadas por outros materiais [1].

A grande variedade de aplicações para as cerâmicas porosas tem motivado o desenvolvimento de diversos processos de fabricação para estes materiais, dentre os quais se destaca: réplica de espuma poliméricas [2], incorporação de fases orgânicas que se volatilizam durante a queima [3] e a decomposição térmica de fases inorgânicas.

Neste trabalho foi utilizado um outro método, desenvolvido pelo Grupo de Engenharia de Microestrutura de Materiais (GEMM-DEMa-UFSCar), no qual os poros são incorporados através da adição de uma espuma aquosa à suspensão cerâmica. A gelificação da suspensão é baseada na 
utilização do alginato de sódio com o objetivo de se formar um gel físico precursor, seguido da adição de diacetato de alumínio hidratado o qual proporciona a formação de ligações químicas entre as moléculas de alginato. Esse método de consolidação tem a vantagem de proporcionar uma rápida solidificação e alta resistência mecânica a verde, além de não utilizar substâncias tóxicas e não ser sensível a presença de oxigênio $[4,5]$.

Com relação à técnica de incorporação de poros, por meio da adição de espumas preparadas isoladamente à suspensão, esta permite maior controle da distribuição e do tamanho dos poros, além de possibilitar que esses dois sistemas, espuma e suspensão, sejam avaliados separadamente facilitando a otimização de ambos.

Tais características representam um avanço tecnológico significativo na produção de cerâmicas porosas uma vez que permite obter maior porosidade associada a um reduzido tamanho de poro. Contudo, o desempenho dos materiais produzidos com essa técnica pode ser ainda superior com o aumento da porosidade, o controle da distribuição do tamanho de poro e da resistência mecânica. Entretanto, estes aspectos dependem das características da suspensão como viscosidade [4], densidade e concentração de sólidos e não somente das característica da espuma.

Dentro deste contexto, esse trabalho teve como objetivo avaliar como as características da suspensão interferem nas propriedades finais das cerâmicas porosas. O tipo de matéria-prima, o método de homogeneização e o teor de sólidos foram os parâmetros selecionados.

\section{EXPERIMENTAL}

\section{Materiais}

Para a preparação das suspensões cerâmicas foram utilizadas as aluminas de alta pureza: A1000SG e A17NE, $>99 \% \mathrm{Al}_{2} \mathrm{O}_{3}$ (Almatis, USA), com tamanho médio de partícula $\left(D_{50}\right)$ de $0,7 \mu \mathrm{m}$ e $4,0 \mu \mathrm{m}$, respectivamente. $\mathrm{O}$ aditivo citrato de diamônio, DAC (Sigma Aldrich), foi utilizado como dispersante para as suspensões de alumina.

A espuma utilizada para incorporar poros foi preparada utilizando o surfactante aniônico de olefina sulfatada (Chemyunion), uma mistura de óleos estabilizantes (Chemyunion) e um agente viscosificante a base de celulose (Degussa). Para a consolidação da cerâmica porosa utilizouse o alginato de sódio (Fluka) com uma distribuição de pesos moleculares variando entre 48000 e $186000 \mathrm{~g} / \mathrm{mol}_{(}\left(\mathrm{M}_{\mathrm{w}}\right)$ e o diacetato de alumínio hidratado, HADA (Sigma Aldrich). A obtenção da cerâmica porosa foi constituída de três etapas: preparação da suspensão, da espuma e da adição dos agentes ligantes.

As suspensões foram preparadas com diferentes características a fim de avaliar a influência da matéria-prima e teor de sólidos nas propriedades finais das cerâmicas porosas. Para avaliação quanto ao tipo de matéria-prima utilizou-se:
1) A $1000 \mathrm{SG}$ ou uma mistura de A17NE com A1000SG, com teor de sólidos de $80 \%$-p; homogeneizadas em moinho de bolas, 2) em relação ao método de homogeneização usouse uma mistura de A17NE com A1000SG, com teor de sólidos de 80\%-p., homogeneizadas em moinho de bolas ou misturador mecânico e, 3) na avaliação quanto ao teor de sólidos usou-se uma mistura de A17NE com A1000SG, com teores de sólidos de 30, 70, 75, 80 e 85\%-p, homogeneizadas em misturador mecânico.

Independente da composição, a preparação das suspensões envolveu a adição à água dos materiais previamente misturados a seco: alumina, alginato e DAC (Tabela I) e a homogeneização realizou-se em misturador mecânico (Ética com $50 \%$ de rotação), seguida ou não de uma homogeneização adicional em moinho de bolas. Neste caso, utilizou-se $50 \%$ em peso de meios de moagem de alumina e tempo de agitação de $14 \mathrm{~h}$, para a completa desaglomeração dos pós.

Separadamente das suspensões foram preparadas as espumas por meio da mistura do surfactante (olefina sulfatada) e água seguida de agitação vigorosa, com o auxílio de uma hélice espiral acoplada a um misturador mecânico para a incorporação da maior quantidade de ar possível. Visando aumentar a estabilidade da espuma gerada foi adicionada uma mistura de óleos estabilizantes e um agente viscosificante a base de celulose, cujos teores (Tabela I) e o procedimento de mistura foram previamente determinado. Em seguida a espuma estável foi adicionada à suspensão cerâmica e homogeneizada através de agitação.

Para a consolidação do sistema (suspensão + espuma) foi utilizado diacetato de alumínio hidratado (HADA) em todas as suspensões preparadas. O HADA foi primeiramente

Tabela I- Reagentes utilizados na preparação da cerâmica porosa.

[Table I - Porous ceramics additives and compositions.]

\begin{tabular}{|c|c|c|c|}
\hline & \multicolumn{2}{|c|}{ Reagentes } & Quantidade (\%p.) \\
\hline \multirow{5}{*}{ 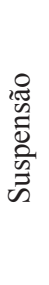 } & \multirow{2}{*}{\multicolumn{2}{|c|}{$\begin{array}{c}\text { A1000SG } \\
\text { A17NE + A1000SG }\end{array}$}} & 100 \\
\hline & & & $93+7$ \\
\hline & \multirow[t]{2}{*}{$\mathrm{DAC}$} & A1000SG & 0,22 \\
\hline & & $\mathrm{A} 17 \mathrm{NE}+\mathrm{A} 1000 \mathrm{SG}$ & 0,11 \\
\hline & Água & $\begin{array}{c}30,70,75 \\
80,85 \% \text {-p sol. }\end{array}$ & $\begin{array}{l}70,30,25 \\
20,15\end{array}$ \\
\hline \multirow{4}{*}{ 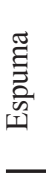 } & \multicolumn{2}{|c|}{ Surfactante } & 0,4 \\
\hline & \multicolumn{2}{|c|}{ Água } & 4 \\
\hline & \multicolumn{2}{|c|}{ Óleos estabilizantes } & 4 \\
\hline & \multicolumn{2}{|c|}{ Viscosificante } & 0,2 \\
\hline 華 & \multicolumn{2}{|c|}{ Alginato de sódio } & 0,15 \\
\hline$\sqrt{30}$ & \multicolumn{2}{|c|}{ HADA } & 0,4 \\
\hline
\end{tabular}

Obs: Os cálculos das quantidades dos reagentes foram baseados na quantidade de alumina 
dissolvido em água e adicionado ao sistema (suspensão + espuma) de acordo com a quantidade indicada na Tabela I.

Depois de incorporada a espuma à suspensão através de agitação, o sistema (suspensão + espuma) foi vertido em moldes previamente lubrificados com um desmoldante. Nesta etapa o sistema sofreu uma pequena vibração para que as bolhas maiores pudessem ser eliminadas.

Todas as amostras foram secas por $12 \mathrm{~h}$ ao ar e mais 12 $\mathrm{h}$ em estufa a $50{ }^{\circ} \mathrm{C}$. Em seguida, foram queimadas em três diferentes temperaturas $1100{ }^{\circ} \mathrm{C}, 1300{ }^{\circ} \mathrm{C}$ e $1500{ }^{\circ} \mathrm{C}$, com taxa de aquecimento de $2{ }^{\circ} \mathrm{C} / \mathrm{min}$, patamar de $5 \mathrm{~h}$ e taxa de resfriamento de $10{ }^{\circ} \mathrm{C} / \mathrm{min}$.

As amostras preparadas a partir de suspensões contendo matérias-primas distintas foram caracterizadas quanto a porosidade, distribuição de tamanho de poros, retração linear e resistência mecânica. Medidas de porosidade e resistência mecânica também foram realizadas para as amostras nas quais o método de homogeneização das suspensões foi variado. Já os corpos preparados com suspensões contendo diferentes teores de sólidos foram caracterizados somente quanto a porosidade, enquanto as suspensões foram submetidas a medidas de viscosidade utilizando-se o reômetro rotativo RS 300 (Thermo Haake). O volume antes e após a adição da espuma foi avaliado com o auxílio de uma proveta.

Os ensaios de porosidade nas amostras obtidas foram realizados pelo método de Arquimedes utilizando-se querosene como líquido de imersão. A distribuição de tamanho de poros foi medida por meio do ensaio de porosimetria de mercúrio (Micromeritics, modelo 9320). A retração linear foi obtida pela diferença entre as dimensões das amostras antes e após a queima; a resistência mecânica foi avaliada por meio de ensaios de compressão diametral (Norma ASTM C496-90) utilizando um equipamento de ensaios universal MTS $810 \mathrm{com}$ taxa de aplicação de carga constante $(11 \mathrm{~N} / \mathrm{s})$. Os resultados dos ensaios de compressão representam uma média de 5 corpos-de-prova para cada ponto, e para a análise de reprodutibilidade, por meio da estatística de Weibull, foram utilizadas 30 amostras.

\section{RESULTADOS E DISCUSSÃO}

\section{Avaliação quanto ao tipo de matéria-prima}

Em geral os processos de fabricação de cerâmicas porosas proporcionam compactos com baixa resistência a verde, microestruturas com grande quantidade de trincas ou ainda poros com geometrias complexas que proporcionam regiões de concentração de tensão. Tais características diminuem a resistência mecânica do produto final impondo sérias restrições à sua utilização em aplicações estruturais. Para melhorar a resistência mecânica é importante densificar a estrutura cerâmica, envolvendo os poros. A seleção de um tipo de matéria-prima com adequada distribuição granulométrica, para a preparação da suspensão, permite maximizar o empacotamento das partículas. Dessa forma, pelo fato da distribuição granulométrica determinar a densidade de empacotamento das partículas, esta tem um efeito marcante na porosidade do corpo conformado e, com isso, sobre a resistência mecânica do produto cerâmico.

O empacotamento ideal de partículas foi descrito por Andreasen como aquele em que, ao redor de duas partículas específicas com tamanhos distintos, há condições de similaridade: isto é, a "imagem da granulação" de ambos os ambientes deve ser a mesma, como se fotografias da vizinhança de cada uma das partículas, quando devidamente ampliadas, fossem as mesmas em todo o sistema de partículas [6]. Essa condição de similaridade define a distribuição de tamanho de partículas em termos de uma lei de potências:

$$
\mathrm{CPFT}=\left(\frac{D_{P}}{D_{L}}\right)^{q} \times 100
$$

onde, $D_{P}$ é o diâmetro da partícula, $D_{L}$ é o diâmetro da maior partícula e $q$ é o módulo ou coeficiente da distribuição.

A distribuição de Andreasen com coeficiente de distribuição, $\mathrm{q}=0,37$, é a de máximo empacotamento teoricamente possível. No entanto, isso ocorre quando o diâmetro da menor partícula é 0 , o que na prática, não se verifica. Sendo assim, para maximizar o empacotamento em sistemas reais, deve-se utilizar o menor $\mathrm{D}_{\mathrm{S}}$ possível [6]. Neste contexto, foram selecionadas as matérias-primas A1000SG e A17NE sendo que a distribuição granulométrica desta última foi otimizada utilizando-se o software PSDesigner resultando numa mistura de A17NE e A1000SG, mencionado no trabalho como A17NEC.

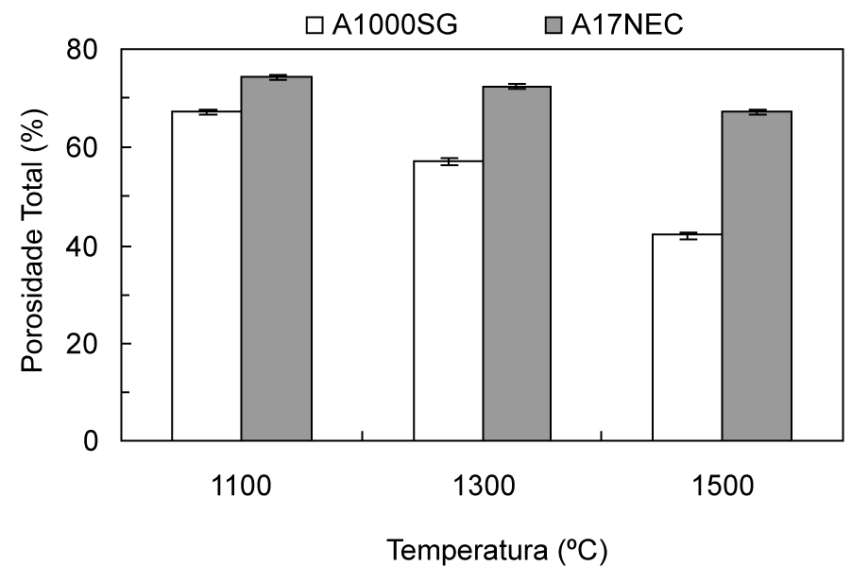

Figura 1: Variação da porosidade total em função da temperatura de queima das cerâmicas porosas obtidas a partir de suspensões de A1000SG ou A17NEC com 80\%-p de sólidos, homogeneizadas em moinho de bolas.

[Figure 1: Total porosity as a function of firing temperature for porous ceramics prepared with A1000SG or A17NEC suspensions (80 wt\% solids), homogenized trough ball milling.] 
Os resultados quanto à porosidade total obtidos para os corpos preparados com as diferentes matérias-primas são apresentados na Fig. 1. Independentemente da temperatura de queima os corpos preparados com a alumina A17NEC apresentaram superior porosidade, sendo que uma diferença de 30\% em volume foi obtida após sinterização na temperatura de $1500^{\circ} \mathrm{C}$. Uma vez que a espuma incorporada às suspensões foi a mesma, tal diferença pode ser atribuída ao empacotamento das partículas como conseqüência de suas distribuições granulométricas. Entretanto, a composição A17NE+A1000SG foi planejada visando um empacotamento superior quando comparado ao uso de A1000SG e, portanto deveria resultar em menor porosidade.

De fato, os resultados quanto às curvas de freqüência de distribuição de poros (Fig. 2) mostram que as amostras preparadas com A17NEC apresentam uma considerável diminuição de poros de diâmetros abaixo de $0,5 \mu \mathrm{m}$, os quais são característicos de compactos bem empacotados. Portanto, outros fatores devem estar influenciando nesta propriedade das cerâmicas porosas como será discutido adiante.

Além disso, observa-se que os poros pequenos $(0,15 \mu \mathrm{m})$
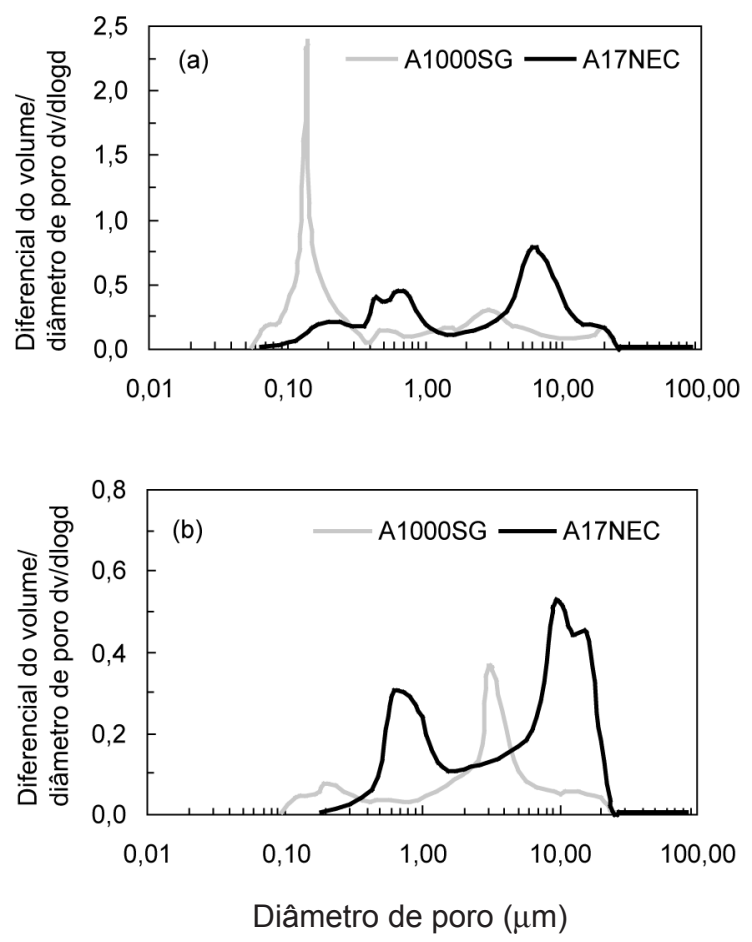

Figura 2: Curvas de freqüência de distribuição de poros das cerâmicas porosas obtidas a partir de suspensões de A1000SG ou A17NEC com 80\%-p. de sólidos, homogeneizadas em moinho de bolas e queimadas a (a) $1100{ }^{\circ} \mathrm{C}$ e (b) $1500{ }^{\circ} \mathrm{C}$.

[Figure 2: Pore size distribution curves for porous ceramics prepared with A1000SG or A17NEC suspensions ( $80 \mathrm{wt} \%$ solids), homogenized trough ball milling and fired at (a) $1100{ }^{\circ} \mathrm{C}$ and (b) $\left.1500{ }^{\circ} \mathrm{C}.\right]$

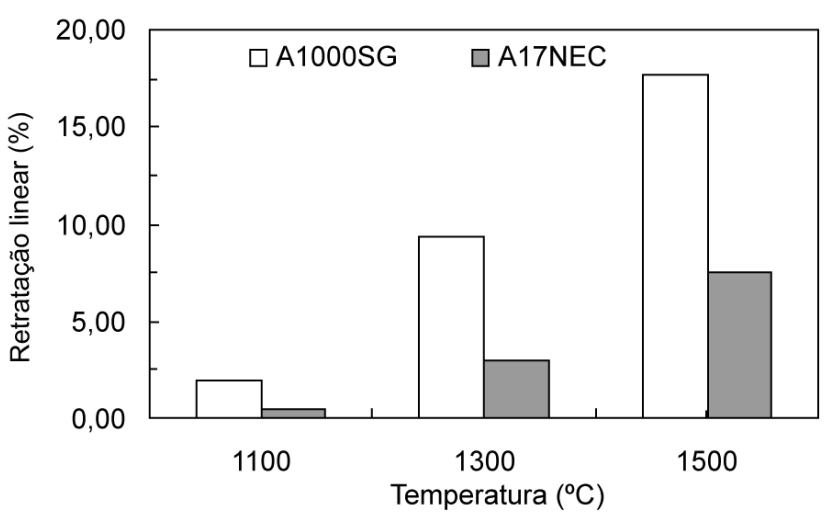

Figura 3: Variação da retração linear em função da temperatura de queima das cerâmicas porosas obtidas a partir de suspensões de A1000SG ou A17NEC com 80\%-p. de sólidos, homogeneizadas em moinho de bolas.

[Figure 3: Linear shrinkage as a function of firing temperature for porous ceramics prepared with A1000SG or A17NEC suspensions (80 wt\% solids), homogenized trough ball milling.]

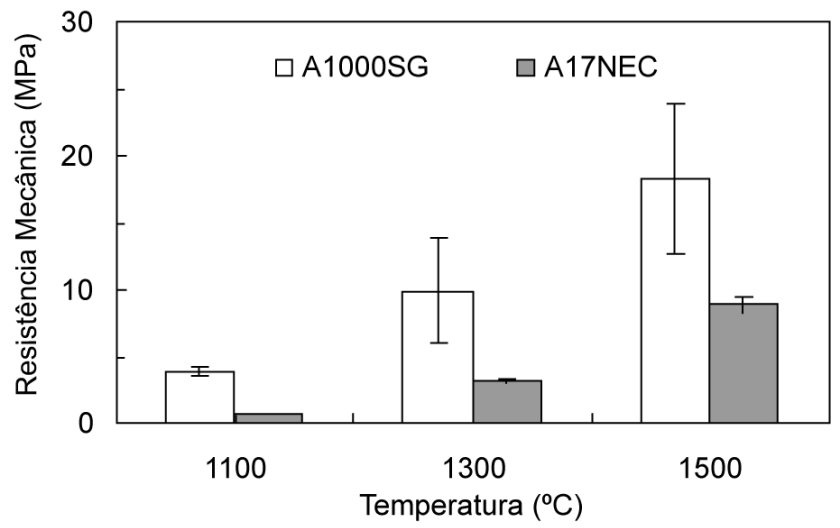

Figura 4: Variação da resistência mecânica em função da temperatura de queima das cerâmicas porosas obtidas a partir de suspensões de A1000SG ou A17NEC com 80\%-p. de sólidos, homogeneizadas em moinho de bolas.

[Figure 4: Mechanical strength as a function of firing temperature for porous ceramics prepared with A1000SG or A17NEC suspensions (80 wt\% solids), homogenized trough ball milling.]

das amostras preparadas com A1000SG desapareceram quando estas foram queimadas a $1500{ }^{\circ} \mathrm{C}$, (Fig. 2 b) resultando numa retração em torno de $10 \%$ maior dessas cerâmicas porosas, quando comparado as amostras com A17NEC (Fig. 3). Consequentemente, a maior retração das amostras preparadas com A1000SG aliada à sua menor porosidade total resultaram numa superior resistência mecânica (Fig. 4).

Quanto à análise de reprodutibilidade nos ensaios de resistência mecânica, observa-se na Fig. 5 que as amostras preparadas com A1000SG apresentaram uma menor reprodutibilidade $(m=6,3)$. Os valores observados para os parâmetros de Weibull, $m$, dependem fortemente do 
processamento. Tipicamente $m$ apresenta valores entre 3 e 15 , sendo que quanto menor o valor de $m$, maior é o espalhamento dos valores de resistência mecânica [7]. Dessa forma, uma vez que as cerâmicas porosas necessitam apresentar alta porosidade foi selecionada a alumina A17NEC para dar continuidade ao trabalho. O uso desta matéria-prima resultou em uma porosidade 30\% superior quando comparado ao da A1000SG, sendo este aumento mais significativo que à perda de resistência mecânica (10\%), além de possibilitar resultados mais confiáveis.

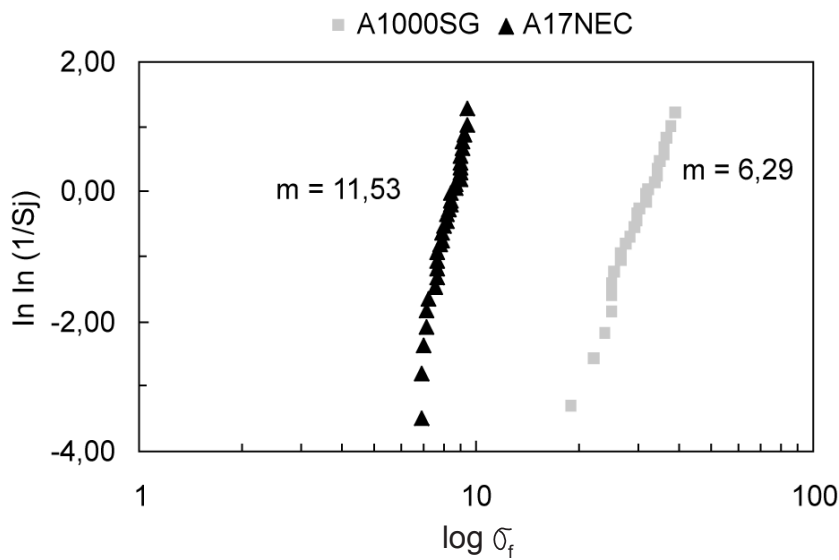

Figura 5: Análise de reprodutibilidade das cerâmicas porosas obtidas a partir de suspensões de A1000SG ou A17NEC com 80\%p. de sólidos, homogeneizadas em moinho de bolas e queimadas à $1500^{\circ} \mathrm{C}$.

[Figure 5: Weibull plot for porous ceramics prepared with A1000SG or A17NEC suspensions ( $80 \mathrm{wt} \%$ solids), homogenized trough ball milling and fired at $1500{ }^{\circ} \mathrm{C}$.]

Avaliação quanto ao método de homogeneização da suspensão

O método de homogeneização utilizado na seção anterior, na preparação das cerâmicas porosas, foi o em moinho de bolas. Este método permite a obtenção de uma suspensão mais homogênea uma vez que fornece energia por meio de impacto e atrito para a quebra de aglomerados de partículas, além de utilizar um tempo longo para a mistura das suspensões. Contudo, a homogeneização utilizando apenas um misturador mecânico proporciona uma redução em termos de tempo de mistura e gasto de energia. Por isso, nesta etapa foi avaliada a influência do método de homogeneização nas propriedades de cerâmicas porosas preparadas com a alumina A17NEC, uma vez que esta proporcionou melhores propriedades como apresentado anteriormente.

Os resultados quanto à porosidade total e resistência mecânica obtidos para os corpos preparados utilizando-se os diferentes métodos de homogeneização são apresentados nas Figs. 6 e 7, respectivamente. Observa-se que as cerâmicas porosas apresentaram valores muito próximos tanto de porosidade como de resistência mecânica para as três temperaturas de queima utilizadas (1100, $1300 \mathrm{e}$ $\left.1500{ }^{\circ} \mathrm{C}\right)$. Tal fato mostrou ser possível alterar o método de homogeneização sem grandes prejuízos às propriedades das cerâmicas porosas.

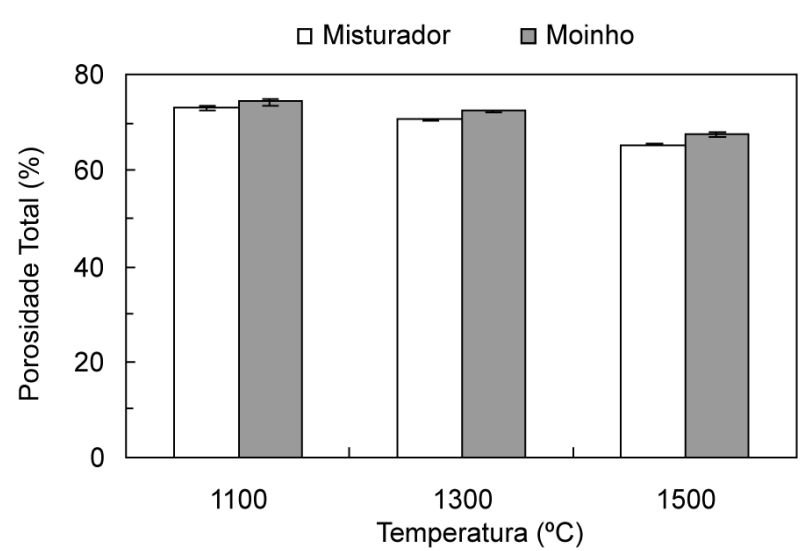

Figura 6: Variação da porosidade total em função da temperatura das cerâmicas porosas obtidas a partir de suspensões de A17NEC com $80 \%$-p. de sólidos, homogeneizadas em misturador ou moinho de bolas.

[Figure 6: Total porosity as a function of firing temperature for porous ceramics prepared with A17NEC suspensions (80 wt\% solids), homogenized trough mechanical mixing or ball milling.]

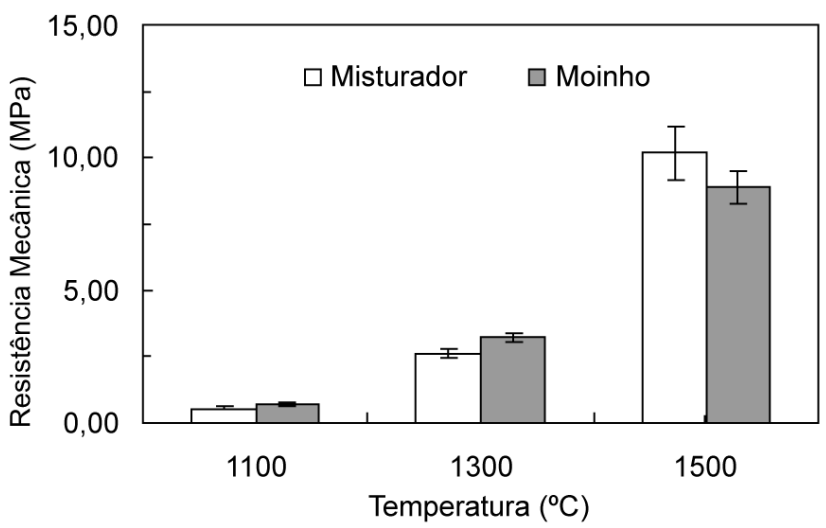

Figura 7: Variação da resistência mecânica em tunção da temperatura das cerâmicas porosas obtidas a partir de A17NEC com 80\%-p. de sólidos, homogeneizados em misturador ou moinho de bolas. [Figure 7: Mechanical strength as a function of firing temperature for porous ceramics prepared with A17NEC suspensions (80 wt\% solids), homogenized trough mechanical mixing or ball milling.]

\section{Avaliação quanto ao teor de sólidos das suspensões}

A viscosidade relativa de suspensões de partículas esféricas depende diretamente da concentração de sólidos. No entanto, esta relação é válida apenas para suspensões bastante diluídas, onde as partículas se comportam como unidades independentes que não interagem entre si [6]. 
Já em suspensões concentradas, além do teor de sólidos, outras características como estado de dispersão e distribuição de tamanho de partículas influenciam a viscosidade, através da alteração da concentração máxima de sólidos $\left(\mathrm{V}_{\mathrm{Sm}}\right)$. Dessa forma, suspensões contendo partículas aglomeradas entre si e/ou com distribuição granulométrica estreita apresentam reduzido valor de $\mathrm{V}_{\mathrm{Sm}}$ e, consequentemente, maior viscosidade relativa. Em contrapartida, suspensões adequadamente dispersas e constituídas por partículas com uma extensa faixa granulométrica apresentam inferior viscosidade relativa em decorrência do seu superior valor de $\mathrm{V}_{\mathrm{Sm}}[6]$.

Assim sendo, as suspensões preparadas com A17NEC, dispersas com $0,31 \mathrm{mg} / \mathrm{m}^{2} \mathrm{DAC}$, contendo diferentes teores de sólidos (30, 70, 75, 80 e 85\%-p.) apresentaram aumento da viscosidade em função do teor de sólidos (Fig. 8). As amostras de cerâmicas porosas obtidas através dessas suspensões apresentaram menor porosidade à medida que o teor de sólidos foi aumentado de 70 até 85\%-p. (Fig. 8).

Afim de entender porque a preparação de cerâmicas porosas a partir de suspensões mais concentradas resultou em corpos menos porosos, foram medidos os volumes das espumas, das suspensões e da mistura espuma mais suspensão, como apresentado na Tabela II. Nesta tabela pode-se perceber que para os teores de 70, 75, 80 e 85\%-p. o volume medido após agitação da mistura (espuma + suspensão) é significativamente menor que o volume calculado para esta mistura, ou seja, pela soma dos volumes de espuma e de suspensão isoladamente.

Diante desses resultados pode-se afirmar que a viscosidade das suspensões apresenta uma influência significativa no preparo das cerâmicas porosas. $\mathrm{O}$ aumento da viscosidade dificulta a homogeneização da espuma na suspensão promovendo o colapso das bolhas e consequentemente o decréscimo da porosidade das cerâmicas porosas obtidas a partir dessa suspensão (Fig. 8). Por outro lado, a suspensão com viscosidade muito baixa como verificado para o teor

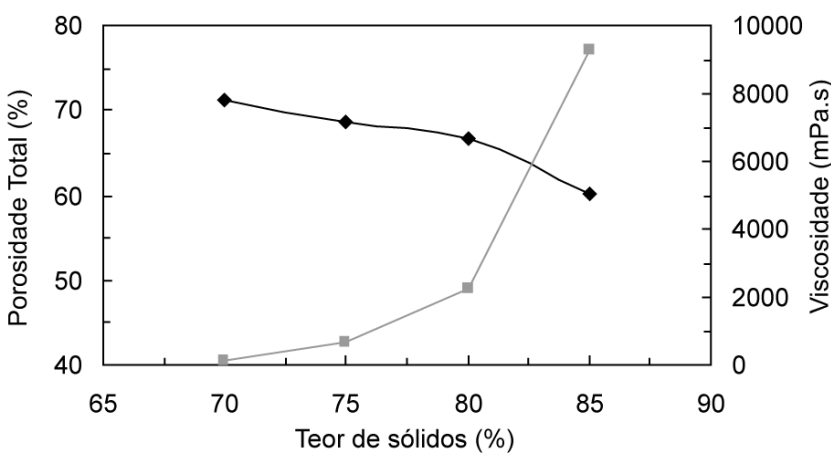

Figura 8: Variação da porosidade total de cerâmicas porosas obtidas a partir de suspensões de A17NEC, dispersas com $0,31 \mathrm{mg} / \mathrm{m}^{2} \mathrm{DAC}$ e da viscosidade das suspensões em função do teor de sólidos, homogeneizadas em misturador mecânico.

[Figure 8: Total porosity for porous ceramics prepared with A17NEC suspensions $\left(0.31 \mathrm{mg} / \mathrm{m}^{2}\right.$ of DAC) and their viscosity as a function of solids content (mechanical mixing).]
Tabela II - Volumes das espumas $\left(\mathrm{V}_{\text {esp }}\right)$, das suspensões $\left(\mathrm{V}_{\text {susp }}\right)$ e da mistura espuma + suspensão $\left(\mathrm{V}_{\text {esp+susp. }}\right)$.

[Table II - Foam volume $\left(V_{\text {esp }}\right)$, suspensions volume $\left(V_{\text {susp }}\right)$, and foam + suspension volume $\left.\left(V_{\text {esp }+ \text { susp }}\right).\right]$

\begin{tabular}{cccccc}
\hline $\begin{array}{c}\text { Teor de } \\
\text { sólidos } \\
(\%-p .)\end{array}$ & $\begin{array}{c}\mathrm{V}_{\text {esp. }} \\
(\mathrm{mL})\end{array}$ & $\begin{array}{c}\mathrm{V}_{\text {susp }} \\
(\mathrm{mL})\end{array}$ & $\begin{array}{c}\mathrm{V}_{\text {esp+susp. }} \\
\text { (medido } \\
\text { após } \\
\text { agitação) } \\
(\mathrm{mL})\end{array}$ & $\begin{array}{c}\mathrm{V}_{\text {esp+susp. }} \\
\text { (calculado) } \\
(\mathrm{mL})\end{array}$ & $\begin{array}{c}\mathrm{V}_{\text {esp+susp. }} \\
\text { (medido } \\
\text { calculado) } \\
(\mathrm{mL})\end{array}$ \\
\hline 30 & 110 & 280 & 550 & 390 & 160 \\
70 & 130 & 70 & 150 & 200 & -50 \\
75 & 120 & 65 & 130 & 185 & -55 \\
80 & 120 & 60 & 100 & 180 & -80 \\
85 & 120 & 55 & 60 & 175 & -115 \\
\hline
\end{tabular}

de sólidos de 30\%-p. (2,8 mPa.s) não forneceu estabilidade suficiente para a espuma levando a separação da água da parte sólida.

Tal relação existente entre a viscosidade e a porosidade permitiu entender porque as cerâmicas porosas preparadas a partir da suspensão de A1000SG apresentaram menor porosidade apesar do empacotamento menos eficiente, como verificado na etapa A. Asuperior iscosidade dessas suspensões (4145 mPa.s) comparado à viscosidade da suspensão de A17NEC (2326 mPa.s), para uma taxa de cisalhamento fixa, resultou no colapso da espuma e consequentemente na redução da porosidade. A menor viscosidade da A17NEC deve-se à sua melhor distribuição granulométrica, já que as duas suspensões possuem o mesmo teor de sólidos (80\%-p.).

\section{CONCLUSÕES}

A porosidade da cerâmica porosa não depende apenas do sistema incorporador de poros, mas também das características da suspensão utilizada. $\mathrm{O}$ uso da alumina A17NEC para o preparo da suspensão mostrou ser mais adequado do que a utilização da A1000SG, resultando em um aumento de $30 \%$ na porosidade do corpo, sem promover um prejuízo significativo da resistência mecânica, além do decréscimo da retração e maior confiabilidade. Já em relação ao método de homogeneização percebeu-se que esse não possui uma influência significativa, permitindo assim que seja utilizado um processo mais simples, o que representa uma redução em termos de tempo e gasto de energia. Quanto ao aumento do teor de sólidos da suspensão, este proporciona um acréscimo da viscosidade que por sua vez afeta a estabilidade da espuma e no decréscimo da porosidade. Embora tenha se observado que suspensões altamente concentradas promovem prejuízo à espuma reduzindo a porosidade das amostras, deve-se utilizar o máximo possível teor de sólidos visando à otimização da resistência mecânica. Neste trabalho verificou-se que esse teor é de $80 \%$-p, acima do qual o efeito da viscosidade é marcante. 


\section{AGRADECIMENTOS}

Os autores agradecem à Almatis - USA pelo fornecimento das matérias-primas, a FAPESP pelo suporte financeiro e ao mestrando R. C. O. Romano pela colaboração durante a realização deste trabalho.

\section{REFERÊNCIAS}

[1] F. S. Ortega, A. E. M. Paiva, J. A. Rodrigues, V. C. Pandolfelli, Cerâmica 49, 309 (2003) 1.

[2] V. R. Salvini, M. D. M. Innocentini, V. C. Pandolfelli,
Am. Ceram. Soc. Bull. 79, 5 (2000) 49.

[3] I. Thijs, J. Luyten, S. Mullens, J. Am. Ceram. Soc. 87, 1 (2003) 170.

[4] F. S. Ortega, P. A. S. Inostroza, V. C. Pandolfelli, Cerâmica 46, 300 (2000) 225.

[5] A. R. Studart, V. C. Pandolfelli, E. Tervoort, J. Am. Ceram. Soc. 85, 11 (2002) 2711.

[6] I. R. de Oliveira, A. R. Studart, R. G. Pileggi, V. C. Pandolfelli, Dispersão e empacotamento de partículas - princípios e aplicações em processamento cerâmico, Fazendo Arte Editorial, S. Paulo, Brasil 1 (2000) 224.

[7] E. D. Zanotto, A. R. Migliore, Cerâmica 37, 247 (1991) 7. (Rec. 09/07/2005, Ac. 11/11/2005) 